Forschende

Komplementärmedizin

Research in

Complementary Medicine
Programmbericht NFP 34

Einleitung

Forsch Komplementärmed 1999;(suppl 1):4-5

\title{
3. Hintergründe und Verlauf des NFP34
}

\author{
P.H. Baumann
}

Im Rahmen der sechsten Serie nationaler Forschungsprogramme beauftragte der Bundesrat am 27. Juni 1990 den Schweizerischen Nationalfonds, einen Ausführungsplan für ein nationales Forschungsprogramm, «Alternative Behandlungsmethoden für Krankheiten des Menschen», abgekürzt «Komplementärmedizin», zu erstellen. Der nationale Forschungsrat ernannte daraufhin eine Expertengruppe, welche am 12. Juni 1991 ihre Arbeit aufnahm und im August einen entsprechenden Ausführungsplan vorlegte. Für das Programm stand ein Rahmenkredit von 6 Millionen Schweizer Franken für fünf Forschungsjahre zur Verfügung.

Wie aber kam es zur Idee dieses Nationalen Forschungsprogramms? In den letzten Jahrzehnten des 20. Jahrhunderts ist sich die Öffentlichkeit der Schweiz, wie auch in der übrigen westlichen Welt, bewusst geworden, das «nichtschulmedizinische» Massnahmen eine ständig wachsende Bedeutung erlangt haben. Das entsprechende Angebot hat sich ausgeweitet, und viele Menschen beanspruchen heute komplementärmedizinische Dienstleistungen. Mediziner und nichtakademische Therapeuten mit alternativer Ausbildung greifen häufig auf komplementärmedizinische Diagnose- und Therapieverfahren zurück. In dieser Situation wurde auch die politische Forderung gestellt, Komplementärmedizin an den medizinischen Hochschulen zu lehren. Daneben haben sich Probleme bezüglich der Entschädigung und Vergütung komplementärmedizinischer Dienstleistungen durch die Krankenversicherungen ergeben. In Anbetracht der Bedeutung, die die Komplementärmedizin in unserer Gesellschaft gewonnen hatte, erschien es den Entscheidungsträgern von allgemeinem Interesse, das Phänomen «Komplementärmedizin» genauer zu analysieren.

Bewegung entstand damals, als der Vorstand der Verbindung der Schweizer Ärzte (FMH) eine Arbeitsgruppe einsetzte (Juli 1988 bis Oktober 1989), die Empfehlungen über mögliche Praxisanwendungen, die Ausbildung der interessierten Ärzte und Fragen der Honorierung erarbeitete. Wichtigstes Resultat war jedoch die Forderung nach adäquater Erforschung des Gebiets im Vergleich zu konventionellen Methoden der Hoch- schulmedizin. In der Folge nahm der Vorstand der Schweizerischen Akademie der Medizinischen Wissenschaften (SAMW, Basel) die Idee auf und schlug dem Bundesrat die Etablierung eines auf nationaler Ebene zu bearbeitenden Forschungsprogrammes vor.

Seit Anfang 1992 gibt es das «Nationale Forschungsprogramm 34, Komplementärmedizin (NFP34)». Die 15 Projekte, die aus über 220 eingereichten Projektvorschlägen zur Ausführung gelangten, behandelten Gebiete, die bisher weder an Universitäten gelehrt wurden noch einen Teil des etablierten Gesundheitswesens darstellten.

Auf drei wesentliche Themenkreise hatte sich die verantwortliche Expertengruppe bei Beginn des Programms festgelegt: 1. Vertiefung der Kenntnisse über die Gründe für die Verbreitung der Komplementärmedizin, 2. Analyse der gesellschaftlichen und wirtschaftlichen Auswirkungen der Komplementärmedizin, und 3. Erarbeitung neuer wissenschaftlicher Methoden zur Beurteilung der Komplementärmedizin.

Entsprechend der dritten Aufgabenstellung umfasste das Spektrum der primär nach methodologischen Gesichtspunkten ausgewählten Forschungsprojekte diejenigen Bereiche und Verfahren, die praxisrelevant und nicht spekulativ waren. Dabei bevorzugte die Expertengruppe bei der Auswahl solche Studien, in denen komplementärmedizinisch tätige Therapeuten mit solchen aus der Schulmedizin in Teams zusammenarbeiteten.

Die in der ersten Phase anonymisiert durchgeführten Beurteilungen der eingereichten Projektvorschläge erfolgte aufgrund folgender Kriterien:

- Übereinstimmung mit den Zielsetzungen des NFP34,

- Fähigkeit, die gestellte Frage (oder Hypothese) zu beantworten,

- Innovationspotential für die Prüfmethodik,

- Allgemeingültigkeit für das betreffende Gebiet (Lehr- und Lernbarkeit),

- Potentielle Bedeutung für das Gesundheitswesen,

- Praktische Durchführbarkeit (realistisches Studiendesign).

\begin{tabular}{ll}
\hline KARGER & $\oplus 1999$ S. Karger GmbH, Freiburg \\
Fax (0761) 4520714 & Accessible online at: \\
$\begin{array}{l}\text { E-mail kargergmbh@aol.com } \\
\text { www.karger.com }\end{array}$ & http://BioMedNet.com/karger
\end{tabular}

Dr. sc. techn. Peter H. Baumann Höhenstrasse 4

CH-4433 Ramlinsburg (Schweiz) 
Die schliesslich nach zum Teil intensiven Gesprächen - in der zweiten Phase - erfolgte Auswahl spiegelt sich in der Liste der Projekttitel. Sozioökonomische Aspekte kommen in 4 Projekten zur Behandlung, medizinisch-klinische in 10. Der Einfluss von Zonen, die unter dem Gesichtspunkt der Radiästhesie aktiv sind, wurde in einem physiologisch orientierten Projekt analysiert.

\section{Wie war der Verlauf des Programms?}

Mit Enthusiasmus und der Bereitschaft, Pionierarbeit zu leisten, starteten 199313 Projektteams ihre Forschungsarbeiten; zwei weitere Projekte wurden in den Folgejahren eröffnet und zwei durch selbständige Fragestellungen erweitert. Allmählich entstand eine grosse Forschungs- und Arbeitsgemeinschaft, deren Ergebnisse und Erfahrungen sowohl in den Einzelberichten (Kapitel 4) als auch in den vielfältigen Schlussfolgerungen (Kapitel 5) zur Sprache kommen. Die wissenschaftlichen Resultate der einzelnen Studien wurden (und werden) von den Projektleitern und ihren Teams in passenden Fachpublikationen unabhängig vom Schweizerischen Nationalfonds vorgestellt und diskutiert (siehe Liste jeweils am Schluss der einzelnen Projektdarstellungen, Kapitel 4; dort finden sich auch die Details zu jedem Projekt).

Die während der ganzen Programmdauer innerhalb der Expertengruppe und ausserhalb in Symposien und Konferenzen zur Sprache gebrachten, grundsätzlichen Fragestellungen sind in einem separaten 6. Kapitel zur Diskussion gestellt. Sie sollten bei weiterführenden Studien in Betracht gezogen werden.

Die Expertengruppe und die Programmleitung führten drei Orientierungs- und Diskussionsveranstaltungen durch:

- 14. Januar 1997 über Komplementärmedizin und Gesundheitsrecht: «Naturheilerinnen und Naturheiler: Zulassen, dulden oder verbieten?» (Projekt: M. Künzi, Gesundheitsrecht Schweiz),

-25. November 1997 über: «Hypnose als Therapie?» (Projekte: T. Gysin, Spannungskopfschmerzen; E. Buchser, Chronische Schmerzen; A. Bircher, Heuschnupfen),
- 12. November 1998 über: «Komplementärmedizin in der Krankenversicherung; Patientenverhalten, Nutzen und Kosten» (Projekte: J. Sommer, Gesundheitsökonomische Analyse; A. Schär und V. Messerli, Patientenverhalten).

Zwei Publikationen orientierten über die Programmanlage bzw. über die Projektinhalte:

- «Portrait NFP34» (28 Seiten), Februar 1994; Herausgeber Nationalfonds, Programmleitung,

- Schweizerische Medizinische Wochenschrift Supplementum 62 vom 30. Juli 1994. Editorial von F.J. Frey; 13 Beiträge der Projektverantwortlichen (94 Seiten) [Schweiz Med Wochenschr Suppl 1994;62].

Die Programmleitung liess sich in vier Beiträgen zum NFP34 vernehmen:

- Neue Züricher Zeitung vom 20./21. November 1993 (Zeitfragen) (p. 25),

- Schweizerische Rundschau für Medizin, PRAXIS, Sondernummer 51/52 [Schweiz Rundsch Med Prax 1994;83: 1432-1438],

- Medicus Mundi, April 1996, p. 6-8,

- Schweizerische Rundschau für Medizin, PRAXIS, Sondernummer 29/30; [Schweiz Rundsch Med Prax 1998;87:954-957].

\section{Literatur zu anderen Komplementärmedizin- Programmen oder Methodendiskussionen}

Andritzky W: Alternative Gesundheitskultur (Eine Bestandesaufnahme mit Teilnehmerbefragung). Berlin, VWB-Verlag für Wissenschaft und Bildung, 1997. European Commission Brüssel (ed): COST Action B4, Unconventional Medicine, Final Report of the Management Committee 1993-1998. EUR 18420(EN), 1998, ISBN 92-828-4672-5.

Hornung J (Hrsg): Forschungsmethoden in der Komplementärmedizin (Ueber die Notwendigkeit einer methodologischen Erneuerung). Stuttgart, Schattauer, 1996.

Melchart D, Wagner H: Naturheilverfahren, Grundlagen einer autoregulativen Medizin (Münchner Modell). Stuttgart, Schattauer, 1993.

Unkonventionelle Medizinische Richtungen (Band 21, FDG, Bundesministerium für Forschung und Technologie). Bremerhaven, Verlag für neue Wissenschaft, 1992. 